\title{
Asymptotic Behavior of the Global Attractors to the Boussinesq System for Rayleigh-Bénard Convection at Large Prandtl Number
}

\author{
Xiaoming Wang \\ Department of Mathematics and Geophysical Fluid Dynamics Institute \\ Florida State University \\ Tallahassee, FL 32306 \\ wxm@math.fsu.edu
}

August 18, 2005

\begin{abstract}
We study asymptotic behavior of the global attractors to the Boussinesq system for Rayleigh-Bénard convection at large Prandtl number. In particular, we show that the global attractors to the Boussinesq system for Rayleigh-Bénard convection converge to that of the infinite Prandtl number model for convection as the Prandtl number approaches infinity. This offers partial justification of the infinite Prandtl number model for convection as a valid simplified model for convection at large Prandtl number even in the long time regime.
\end{abstract}

keywords: Rayleigh-Bénard convection, Boussinesq equations, Prandtl number, infinite Prandtl number model, Rayleigh number, global attractor, suitable weak solution

\section{Introduction}

One of the fundamental systems in fluid dynamics is the following Boussinesq system for Raleigh-Bénard convection (non-dimensional):

$$
\frac{1}{P r}\left(\frac{\partial \mathbf{u}}{\partial t}+(\mathbf{u} \cdot \nabla) \mathbf{u}\right)+\nabla p=\Delta \mathbf{u}+R a \mathbf{k} T, \quad \nabla \cdot \mathbf{u}=0,
$$




$$
\begin{aligned}
\frac{\partial T}{\partial t}+\mathbf{u} \cdot \nabla T & =\Delta T \\
\left.\mathbf{u}\right|_{z=0,1}= & 0, \\
\left.T\right|_{z=0}=1, & \left.T\right|_{z=1}=0, \\
\left.\mathbf{u}\right|_{t=0}=\mathbf{u}_{0}, & \left.T\right|_{t=0}=T_{0},
\end{aligned}
$$

where $\mathbf{u}$ is the fluid velocity field, $p$ is the modified pressure, $T$ is the temperature field and $\mathbf{k}$ is the unit upward vector.

The system is a model for convection, i.e., fluid motion induced by differential heating, of a layer of fluids bounded by two horizontal parallel plates a distance $h$ apart in the Rayleigh-Bénard setting ([39], [20]) with the bottom plate heated at the temperature $T_{2}$ and the top plate cooled at the temperature $T_{1}\left(T_{1}<T_{2}\right)$.

We assume that he fluids occupy the (non-dimensionalized) region

$$
\Omega=\left[0, L_{x}\right] \times\left[0, L_{y}\right] \times[0,1]
$$

with periodicity in the horizontal directions assumed for simplicity. The parameters of the system are thus absorbed into the geometry of the domain plus two non-dimensional numbers: the Rayleigh number

$$
R a=\frac{g \alpha\left(T_{2}-T_{1}\right) h^{3}}{\nu \kappa}
$$

measuring the ratio of overall buoyancy force to the damping coefficients; and the Prandtl number

$$
\operatorname{Pr}=\frac{\nu}{\kappa}
$$

measuring the relative importance of kinematic viscosity over thermal diffusivity. Here $\nu$ and $\kappa$ are the kinematic viscosity and thermal diffusive coefficient respectively, $\alpha$ is the thermal expansion coefficient of the fluid, $g$ is the gravitational constant, $h$ is the distance between the two plates confining the fluid, $T_{2}-T_{1}$ is the temperature difference between the bottom and top plates, and we have taken the distance between the plates, $h$, as typical length scale, the thermal diffusive time as typical time scale, and the temperature is scaled so that the top plate is set to 0 while the bottom plate is set to 1 (see [39] among others).

The Boussinesq system exhibits extremely rich phenomena from pure conduction at low Rayleigh number, to Bénard cells at first bifurcation, spatialtemporal patterns and chaos at intermediate Rayleigh number, all the way 

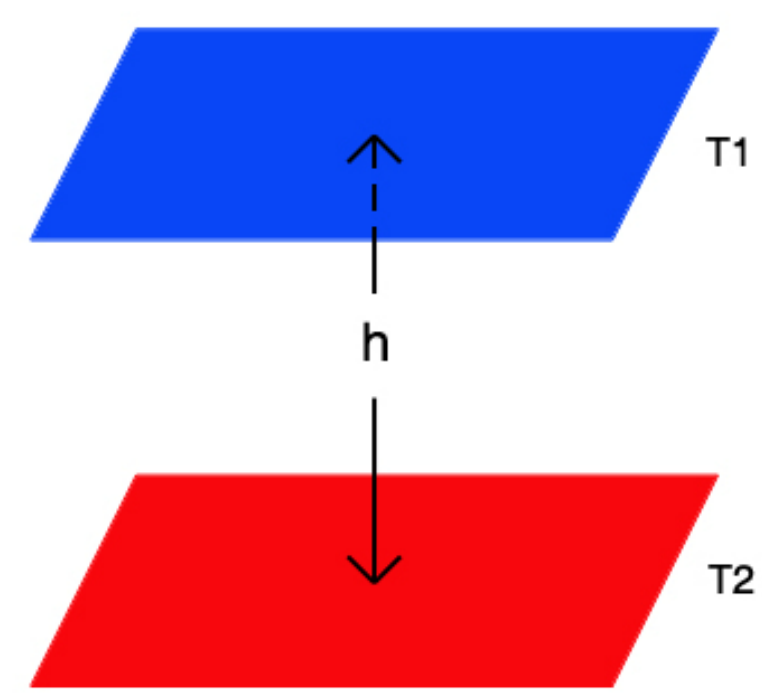

Figure 1: Rayleigh-Bénard convection

to convective turbulence at high Rayleigh number (see for instance [20], [39], and the recent review by [3], [34]). On the other hand, we have very limited mathematical knowledge on the system. Even the issue of the existence of regular enough solutions is unresolved. Indeed, the velocity equation is exactly the Navier-Stokes system (forced by a buoyancy term) whose regularity of solutions is one of the million dollar mathematical problems of the new millennium (www.claymath.org/Millennium_Prize_Problems/). For such a complex system, simplification is highly desirable. Simpler model can be obtained if we consider the regime of large Prandtl number. If we formally set the Prandtl number to infinity, we arrive at the following

infinite Prandtl number model (non-dimensional)

$$
\begin{aligned}
\nabla p^{0} & =\Delta \mathbf{u}^{0}+R a \mathbf{k} T^{0}, \quad \nabla \cdot \mathbf{u}^{0}=0 \\
\frac{\partial T^{0}}{\partial t}+\mathbf{u}^{0} \cdot \nabla T^{0} & =\Delta T^{0} \\
\left.\mathbf{u}^{0}\right|_{z=0,1} & =0 \\
\left.T^{0}\right|_{z=0}=1, & \left.T^{0}\right|_{z=1}=0 .
\end{aligned}
$$

(see for instance [3], [4], [5], [7], [21], [39], [40], [41] among others) which is relevant for fluids such as silicone oil and the earth's mantle as well as many 
gases under high pressure. One observes that the Navier-Stokes equations in the Boussinesq system has been replaced by the Stokes system in the infinite Prandtl number model.

The fact that the velocity field is linearly "slaved" by the temperature field has been exploited in several recent very interesting works on rigorous estimates on the rate of heat convection in this infinite Prandtl number setting (see [10], [7], [9] and the references therein, as well as the work of [4]).

An important natural question is whether such an approximation is valid.

The mathematical justification of the infinite Prandtl number model on a short time interval can be found in [41]. Encouraged by the finite time convergence, we naturally inquire if the solutions of the Boussinesq system and solutions of the infinite Prandtl number model remain close on a large time interval for large Prandtl number. In general we should not expect long time proximity of each individual orbit. Such a long time orbital stability result shouldn't be expected for such complex systems where turbulent/chaotic behavior abound. Instead, the statistical properties for such systems are much more important and physically relevant and hence it is natural to ask if the statistical properties (in terms of invariant measures) as well as global attractors (if they exist) remain close.

The first obstacle in studying long time behavior is the well-posedness of the Boussinesq system global in time. This is closely related to the well-known problem related to the 3D Navier-Stokes equations([8], [37], [28] among others). This is partially resolved by considering suitable weak solutions (see section 2). Indeed, we are able to show the eventual regularity for suitably defined weak solutions to the Boussinesq system which exists for all time [42].

Recall that the global attractor of a given dynamical system is a compact invariant set which attracts all bounded set in the phase space ([18], [36] among others). In particular, the global attractor is maximum in the sense that any compact invariant set must be a subset of the global attractor. The global attractor is also minimum in the sense that any bounded set that attracts arbitrary bounded set in the phase space must contain the global attractor. Therefore the closeness of global attractors, if they exist, would be a good measure of closeness of long time behavior.

Although the dynamics of the Boussinesq system may not be well-defined due to the well-known regularity problem, all properly defined weak solutions become regular after a transitional time at large Prandtl number ([42]). The dynamics is also well-defined if solutions start from a bounded set in a 
(smaller) subspace of the phase space. Moreover, such a bounded set is in fact absorbing in the sense all suitably defined weak solutions will enter this bounded set in finite time ([42]). Furthermore, the system possesses a global attractor which is regular in space and in time, and attracts all suitably defined weak solutions ([42]). It is also known that the infinite Prandtl number model for convection possesses a global attractor. Hence, it makes sense to discuss the closeness of the global attractors.

Another issue we encounter here is the difference in natural phase spaces: for the Boussinesq system we need both velocity and temperature while only the temperature field is needed for the infinite Prandtl number model since the velocity field is linearly slaved. There are two ways of handling the discrepancy in phase space: 1. project the phase space of the Boussinesq system down to the temperature field only; 2 . lift the phase space of the infinite Prandtl number model to the product space of velocity and temperature. We will see that the comparison of global attractors after projection is relatively easy, and is similar to the upper semi-continuity of global attractors for dynamical systems (see for instance [18], [36]). The comparison of global attractors after lifting the phase space of the infinite Prandtl number model is a little bit more involved. Here we view the Boussinesq system as a small perturbation of the infinite Prandtl number model. The proximity of global attractors follows from appropriate a priori estimates (uniform in Prandtl number) on the material derivative of the velocity field after the initial layer. The convergence result (corollary 1) was announced earlier [40, 41].

The rest of the manuscript is organized as follows. In section 2 we derive a few a priori estimates needed in the the proof in section 3. These estimates refine previous estimates [42]. In section 3 we present our main results on the convergence of the global attractors of the Boussinesq system to that of the infinite Prandtl number model. The proof of the main results are sketched as well. In section 4 we make concluding remarks and comments. In particular, we discuss if similar results are valid for other systems with multiple time scales.

Throughout this manuscript, we assume the physically important assumption of domain having large aspect ratio, i.e.,

$$
L_{x} \geq 1, \quad L_{y} \geq 1, \text { and hence } \quad|\Omega| \geq 1 .
$$

Likewise, we also assume the physically important case of high Rayleigh number

$$
R a \geq 1
$$


so that we may have non-trivial dynamics.

We also follow the mathematical tradition of denoting our small parameter as $\varepsilon$, i.e.

$$
\varepsilon=\frac{1}{\operatorname{Pr}}
$$

\section{A Priori Estimates}

Here we establish a few a priori estimates on suitable weak solutions to the Boussinesq system needed in the proof of the convergence of the global attractors. The estimates given here refines previous estimates [42] and are uniform in terms of the (large) Prandtl number, or equivalently, the small parameter $\varepsilon$. They are uniform in time as well modulo an initial layer. An initial time layer has to be neglected here since the time derivative is proportional to $1 / \varepsilon$ within certain initial layer [41]. Estimates in higher order Sobolev spaces can be derived as well.

Following traditional approach, we recast the Boussinesq system in terms of the perturbative variable

$$
(\mathbf{u}, \theta)=(\mathbf{u}, T-(1-z))
$$

(perturbation away from the pure conduction state $(0,1-z)$ ).

Non-dimensional Boussinesq system for Raleigh-Bénard convection in perturbative variable:

$$
\begin{aligned}
\frac{1}{\operatorname{Pr}}\left(\frac{\partial \mathbf{u}}{\partial t}+(\mathbf{u} \cdot \nabla) \mathbf{u}\right)+\nabla p & =\Delta \mathbf{u}+\operatorname{Ra} \mathbf{k} \theta, \quad \nabla \cdot \mathbf{u}=0 \\
\frac{\partial \theta}{\partial t}+\mathbf{u} \cdot \nabla \theta-u_{3} & =\Delta \theta \\
\left.\mathbf{u}\right|_{z=0,1} & =0 \\
\left.\theta\right|_{z=0}=1, & \left.\theta\right|_{z=1}=0 \\
\left.\mathbf{u}\right|_{t=0}=\mathbf{u}_{0}, & \left.\theta\right|_{t=0}=\theta_{0}
\end{aligned}
$$

The infinite Prandtl number model for convection can be casted in terms of the perturbative variable in a similar fashion.

Non-dimensional infinite Prandtl number model in perturbative variable

$$
\nabla p^{0}=\Delta \mathbf{u}^{0}+R a \mathbf{k} \theta^{0}, \quad \nabla \cdot \mathbf{u}^{0}=0
$$




$$
\begin{aligned}
\frac{\partial \theta^{0}}{\partial t}+\mathbf{u}^{0} \cdot \nabla \theta^{0}-u_{3}^{0} & =\Delta \theta^{0} \\
\left.\mathbf{u}^{0}\right|_{z=0,1} & =0 \\
\left.\theta^{0}\right|_{z=0,1} & =0
\end{aligned}
$$

Next, we recall the definition of suitable weak solutions [42]

Definition 1 (Suitable Weak Solution) $(\mathbf{u}, \theta)$ is called a suitable weak solution to the Boussinesq equations on the time interval $\left[0, T^{*}\right]$ with given initial data $\left(\mathbf{u}_{0}, \theta_{0}\right)$ if the following hold

$$
\begin{aligned}
\mathbf{u} & \in L^{\infty}\left(0, T^{*} ; H\right) \cap L^{2}\left(0, T^{*} ; V\right) \cap C_{w}\left(\left[0, T^{*}\right] ; H\right), \\
\mathbf{u}^{\prime} & \in L^{4 / 3}\left(0, T^{*} ; V^{\prime}\right), \mathbf{u}(0)=\mathbf{u}_{0}, \\
\theta \quad & \in L^{\infty}\left(0, T^{*} ; L^{2}(\Omega)\right) \cap L^{2}\left(0, T^{*} ; H_{0}^{1}(\Omega)\right) \cap C_{w}\left(\left[0, T^{*}\right] ; L^{2}(\Omega)\right), \\
\theta^{\prime} & \in L^{4 / 3}\left(0, T^{*} ; H^{-1}(\Omega)\right), \theta(0)=\theta_{0} \\
\left.\varepsilon\left(\frac{d}{d t} \int_{\Omega} \mathbf{u} \cdot \mathbf{v}+\int_{\Omega}(\mathbf{u} \cdot \nabla) \mathbf{u} \cdot \mathbf{v}\right)+\int_{\Omega} \nabla \mathbf{u} \cdot \nabla \mathbf{v}\right) & =R a \int_{\Omega} \theta v_{3}, \forall \mathbf{v} \in V,(30) \\
\frac{d}{d t} \int_{\Omega} \theta \eta+\int_{\Omega} \mathbf{u} \cdot \nabla \theta \eta+\int_{\Omega} \nabla \theta \cdot \nabla \eta & =\int_{\Omega} u_{3} \eta, \forall \eta \in H_{0}^{1}, \\
\frac{\varepsilon}{2} \frac{d}{d t}|\mathbf{u}(t)|_{L^{2}}^{2}+|\nabla \mathbf{u}(t)|_{L^{2}}^{2} & \leq R a \theta(t) u_{3}(t), \\
\frac{d}{d t}|(T-1)+(t)|_{L^{2}}^{2}+2\left|\nabla(T-1)^{+}(t)\right|_{L^{2}}^{2} & \leq 0 \\
\frac{d}{d t}\left|T^{-}(t)\right|_{L^{2}}^{2}+2\left|\nabla T^{-}(t)\right|_{L^{2}}^{2} & \leq 0
\end{aligned}
$$

Here we have used standard notations on function spaces used in the study of incompressible fluids (see for instance [8, 11, 17, 37] among others). In particular, the energy inequalities should be understood in the weak sense with initial value taken into consideration.

Recall our goal here is to derive uniform in large Prandtl number Pr, or small $\varepsilon$ estimates that are also uniform in time after neglecting an initial transitional time interval.

We will start with the easy $L^{2}$ estimates for the temperature and velocity field. In the second stage we derive uniform $H^{1}$ estimates for the solutions. These estimates imply the eventual regularity of solutions to the Boussinesq system for convection and the existence of global attractors [42]. In the third 
stage, we derive uniform estimates on the time derivative of the solutions. In the fourth stage, we utilize the uniform estimates on the time derivative to derive uniform estimates in $H^{2}$.

We start with $L^{2}$ estimates on the temperature field. Thanks to $(33,34)$, and Poincaré inequality, we see that

$$
\begin{aligned}
\left|(T-1)^{+}(t)\right|_{L^{2}}^{2} & \leq e^{-2 t}\left|\left(T_{0}-1\right)^{+}\right|_{L^{2}}^{2} \leq e^{-2 t}\left|T_{0}\right|_{L^{2}}^{2}, \\
\left|T^{-}(t)\right|_{L^{2}}^{2} & \leq e^{-2 t}\left|T_{0}\right|_{L^{2}}^{2} .
\end{aligned}
$$

Therefore,

$$
\begin{aligned}
|T(t)|_{L^{2}} & \leq\left|T^{-}(t)\right|_{L^{2}}+\left|T^{+}(t)\right|_{L^{2}} \\
& \leq\left|T^{-}(t)\right|_{L^{2}}+\left|(T-1)^{+}(t)\right|_{L^{2}}+|\Omega| \\
& \leq 2 e^{-t}\left|T_{0}\right|_{L^{2}}+|\Omega| .
\end{aligned}
$$

Hence, for

$$
\begin{gathered}
t_{1}=\ln \frac{2\left|T_{0}\right|_{L^{2}}}{|\Omega|} \\
|T(t)|_{L^{2}} \leq 2|\Omega|, \quad t \geq t_{1} .
\end{gathered}
$$

With the $L^{2}$ estimate in temperature, the $L^{2}$ estimate of the perturbative variable $\theta$ is obvious. Indeed,

$$
\begin{aligned}
|\theta(t)|_{L^{2}} & \leq|T(t)|_{L^{2}}+|1-z|_{L^{2}} \\
& \leq 2 e^{-t}\left|T_{0}\right|_{L^{2}}+2|\Omega| \\
& \leq 3|\Omega|, \quad \forall t \geq t_{1} .
\end{aligned}
$$

This, together with the energy inequality for the velocity field (32), implies

$$
\frac{\varepsilon}{2} \frac{d}{d t}|\mathbf{u}(t)|_{L^{2}}^{2}+|\nabla \mathbf{u}(t)|_{L^{2}}^{2} \leq R a\left(2 e^{-t}\left|T_{0}\right|_{L^{2}}+2|\Omega|\right)\left|u_{3}(t)\right|_{L^{2}},
$$

which further implies, by Poincaré and Cauchy-Schwarz inequality,

$$
\varepsilon \frac{d}{d t}|\mathbf{u}(t)|_{L^{2}}^{2}+|\mathbf{u}(t)|_{L^{2}}^{2} \leq 4 R a^{2}\left(e^{-t}\left|T_{0}\right|_{L^{2}}+|\Omega|\right)^{2} .
$$

Therefore, by Gronwall inequality,

$$
|\mathbf{u}(t)|_{L^{2}}^{2} \leq\left|\mathbf{u}_{0}\right|_{L_{2}}^{2} e^{-\frac{t}{\varepsilon}}+4 R a^{2}\left(\frac{1}{1-2 \varepsilon} e^{-2 t}\left|T_{0}\right|_{L^{2}}^{2}+\frac{2}{1-\varepsilon} e^{-t}\left|T_{0}\right|_{L^{2}}|\Omega|+|\Omega|^{2}\right)
$$


Hence, for

$$
t_{2}=\max \left\{t_{1}, \frac{1}{2} \ln \left(\frac{\left|\mathbf{u}_{0}\right|_{L^{2}}}{|\Omega| R a}\right), \ln \left(\frac{16\left|T_{0}\right|_{L^{2}}}{3|\Omega|}\right)\right\}
$$

and $\varepsilon<\frac{1}{4}$, we have

$$
|\mathbf{u}(t)|_{L^{2}} \leq 3 R a|\Omega|, \quad \forall t \geq t_{2}
$$

This completes the uniform estimates in the $L^{2}$ space.

Next, we focus on the uniform estimates in the $H^{1}$ space.

We first derive uniform $H^{1}$ bound for the velocity.

Following [42], we show that a ball of radius $R_{1}=c_{1} R a$ is absorbing after time $t_{1}$ for suitable $c_{1}(55)$.

Indeed, multiplying the velocity equation in the Boussinesq equation (17) by $A \mathbf{u}$, where $A$ is the Stokes operator $([8,11,17,37])$, integrating over $\Omega$, applying Cauchy-Schwarz and Agmon's inequality and the uniform estimate on $\theta$ (40), we have, for $t \geq t_{1}$,

$$
\begin{aligned}
\frac{\varepsilon}{2} \frac{d}{d t}|\nabla \mathbf{u}|_{L^{2}}^{2}+|A \mathbf{u}|_{L^{2}}^{2} & \leq R a|\theta|_{L^{2}}|A \mathbf{u}|_{L^{2}}+\varepsilon|\nabla \mathbf{u}|_{L^{2}}|A \mathbf{u}|_{L^{2}}|\mathbf{u}|_{L^{\infty}} \\
& \leq R a|\theta|_{L^{2}}|A \mathbf{u}|_{L^{2}}+c_{2} \varepsilon|\nabla \mathbf{u}|_{L^{2}}^{\frac{3}{2}}|A \mathbf{u}|_{L^{2}}^{\frac{3}{2}} \\
& \leq \frac{1}{2}|A \mathbf{u}|_{L^{2}}^{2}+R a^{2}|\theta|_{L^{2}}^{2}+64 c_{2}^{4} \varepsilon^{4}|\nabla \mathbf{u}|_{L^{2}}^{6} \\
& \leq \frac{1}{2}|A \mathbf{u}|_{L^{2}}+9|\Omega|^{2} R a^{2}+64 c_{2}^{4} \varepsilon^{4}|\nabla \mathbf{u}|_{L^{2}}^{6}
\end{aligned}
$$

Hence,

$$
\varepsilon \frac{d}{d t}|\nabla \mathbf{u}|_{L^{2}}^{2}+|\nabla \mathbf{u}|_{L^{2}}^{2} \leq 18|\Omega|^{2} R a^{2}+128 c_{2}^{4} \varepsilon^{4}|\nabla \mathbf{u}|_{L^{2}}^{6}
$$

Consequently, we have,

$$
\frac{d}{d t}|\nabla \mathbf{u}|_{L^{2}}^{2} \leq 0
$$

provided the following hold simultaneously

$$
\begin{gathered}
\frac{1}{2}|\nabla \mathbf{u}|_{L^{2}}^{2} \geq 18|\Omega|^{2} R a^{2}, \\
\frac{1}{2}|\nabla \mathbf{u}|_{L^{2}}^{2} \geq 128 c_{2}^{4} \varepsilon^{4}|\nabla \mathbf{u}|_{L^{2}}^{6},
\end{gathered}
$$


or equivalently,

$$
\begin{gathered}
|\nabla \mathbf{u}|_{L^{2}}^{2} \geq 36|\Omega|^{2} R a^{2} \\
|\nabla \mathbf{u}|_{L^{2}}^{2} \leq \frac{1}{16 c_{2}^{2} \varepsilon^{2}}
\end{gathered}
$$

Hence we need

$$
36|\Omega|^{2} R a^{2} \leq \frac{1}{16 c_{2}^{2} \varepsilon^{2}}
$$

i.e.

$$
\varepsilon R a=\frac{R a}{\operatorname{Pr}} \leq \frac{1}{24 c_{2}|\Omega|}
$$

This is the exact condition of large Prandtl number that we need as was discovered earlier [42].

Now we set

$$
c_{1}=6
$$

and we observe that the ball of radius

$$
R_{1}=c_{1} R a|\Omega|=6 R a|\Omega|
$$

in $H^{1}$ centered at the origin is invariant for the velocity field after $t_{1}$ under the large Prandtl number assumption (54) since $\frac{d}{d t}|\nabla \mathbf{u}|_{L^{2}}^{2}<0$ at the boundary of the ball for $t \geq t_{1}$.

In order to show that this ball is absorbing, we need to show that the velocity field must enter this ball within a set period of time after $t_{1}$. For this purpose, we go back to the energy inequality for the velocity (41), and we can deduce

$$
\begin{aligned}
& \frac{1}{t-t_{2}} \int_{t_{2}}^{t}|\nabla \mathbf{u}(s)|_{L^{2}}^{2} d s \\
\leq & \frac{\varepsilon}{t-t_{2}}\left|\mathbf{u}\left(t_{2}\right)\right|_{L^{2}}^{2}+\frac{4 R a^{2}}{t-t_{2}}\left(\frac{1}{2}\left|T_{0}\right|_{L^{2}}^{2}+2\left|T_{0}\right|_{L^{2}}|\Omega|+\left(t-t_{2}\right)|\Omega|^{2}\right)
\end{aligned}
$$

For the given $c_{1}=6$, and $\varepsilon<\frac{1}{4}$, we define

$$
t_{3}=\max \left\{t_{2}+\frac{9}{4}, t_{2}+\frac{2\left|T_{0}\right|_{L^{2}}^{2}}{|\Omega|^{2}} \cdot t_{2}+\frac{2\left|T_{0}\right|_{L^{2}}}{|\Omega|}\right\}
$$


We then have

$$
\frac{1}{t-t_{2}} \int_{t_{2}}^{t}|\nabla u(s)|_{L^{2}}^{2} d s \leq c_{1}^{2}|\Omega|^{2} R a^{2} / 2=18|\Omega|^{2} R a^{2}, \quad t \geq t_{3},
$$

which implies the existence of $t^{*} \in\left[t_{2}, t_{3}\right]$, such that

$$
\left|\nabla \mathbf{u}\left(t^{*}\right)\right|_{L^{2}} \leq c_{1}|\Omega| R a=R_{1} .
$$

Therefore,

$$
|\nabla \mathbf{u}(t)|_{L^{2}} \leq 6|\Omega| R a, \quad \forall t \geq t_{3}
$$

Now we see that a ball of radius $R_{1}=c_{1}|\Omega| R a=6|\Omega| R a$ in $H^{1}$ is absorbing for the velocity field.

This uniform estimate in $H^{1}$ for the velocity field implies similar $H^{1}$ estimate for the perturbative temperature field. Indeed, multiplying the temperature equation (18) by $\theta$ and integrating over $\Omega$, applying Poincaré inequality, we have

$$
\frac{d}{d t}|\theta|_{L^{2}}^{2}+|\theta|_{L^{2}}^{2}+|\nabla \theta|_{L^{2}}^{2} \leq 2\left|u_{3}\right|_{L^{2}}|\theta|_{L^{2}} \leq 9 R a|\Omega|^{2} \text {, for } t \geq t_{2} .
$$

This implies that for any $t \geq t^{*} \geq t_{2}$

$$
\begin{aligned}
e^{-t} \int_{t^{*}}^{t} e^{s}|\nabla \theta(s)|_{L^{2}}^{2} d s & \leq 9 R a|\Omega|^{2}+e^{-\left(t-t^{*}\right)}\left|\theta\left(t^{*}\right)\right|_{L^{2}}^{2} \\
& \leq 9 R a|\Omega|^{2}+9 e^{-\left(t-t^{*}\right)}|\Omega|^{2} \\
& \leq 10 R a|\Omega|^{2}
\end{aligned}
$$

We also have, for $t \geq t_{3}+1$,

$$
\begin{aligned}
\frac{1}{t-t_{3}} \int_{t_{3}}^{t}|\nabla \theta(s)|_{L^{2}}^{2} & \leq \frac{\left|\theta\left(t_{3}\right)\right|_{L^{2}}^{2}}{t-t_{3}}+9 R a|\Omega|^{2} \\
& \leq 9|\Omega|^{2}\left(R a+\frac{1}{t-t_{3}}\right) \\
& \leq 10|\Omega|^{2} R a, \text { for } t \geq t_{3}+1 .
\end{aligned}
$$

This implies there exists $t^{*} \in\left[t_{3}, t_{3}+1\right]$, such that

$$
\left|\nabla \theta\left(t^{*}\right)\right|_{L^{2}}^{2} \leq 10|\Omega|^{2} R a .
$$


Next, we multiply the perturbative temperature equation (18) by $-\Delta \theta$ and integrate over $\Omega$, we then have

$$
\begin{aligned}
\frac{1}{2} \frac{d}{d t}|\nabla \theta|_{L^{2}}^{2}+|\Delta \theta|_{L^{2}}^{2} & \leq\left|u_{3}\right|_{L^{2}}|\Delta \theta|_{L^{2}}+|\mathbf{u}|_{L^{6}}|\nabla \theta|_{L^{3}}|\Delta \theta|_{L^{2}} \\
& \leq \frac{1}{4}|\Delta \theta|_{L^{2}}^{2}+\left|u_{3}\right|_{L^{2}}^{2}+c_{3}|\nabla \mathbf{u}|_{L^{2}}|\nabla \theta|_{L^{2}}^{\frac{1}{2}}|\Delta \theta|_{L^{2}}^{\frac{3}{2}} \\
& \leq \frac{1}{2}|\Delta \theta|_{L^{2}}^{2}+\left|u_{3}\right|_{L^{2}}^{2}+c_{4}|\nabla \mathbf{u}|_{L^{2}}^{4}|\nabla \theta|_{L^{2}}^{2} .
\end{aligned}
$$

Hence, after applying Poincaré inequality, we have, for $t \geq t_{3}$,

$$
\begin{aligned}
\frac{d}{d t}|\nabla \theta|_{L^{2}}^{2}+|\nabla \theta|_{L^{2}}^{2} & \leq 2\left|u_{3}\right|_{L^{2}}^{2}+2 c_{4}|\nabla \mathbf{u}|_{L^{2}}^{4}|\nabla \theta|_{L^{2}}^{2} \\
& \leq 18 R a^{2}|\Omega|^{2}+2 c_{4} 6^{4}|\Omega|^{4} R a^{4}|\nabla \theta|_{L^{2}}^{2}
\end{aligned}
$$

This implies, with Gronwall inequality and $t^{*}$ chosen in (65) and the intermediate estimate (63),

$$
\begin{aligned}
|\nabla \theta(t)|_{L^{2}}^{2} & \leq e^{-\left(t-t^{*}\right)}\left|\nabla \theta\left(t^{*}\right)\right|_{L^{2}}^{2}+18 R a^{2}|\Omega|^{2}+20 c_{4} 6^{4}|\Omega|^{6} R a^{5} \\
& \leq 10|\Omega|^{2} R a+18|\Omega|^{2} R a^{2}+20 c_{4} 6^{4}|\Omega|^{6} R a^{5} \\
& \leq c_{5}|\Omega|^{6} R a^{5}, \quad \forall t \geq t_{3}+1 .
\end{aligned}
$$

This uniform $H^{1}$ norm estimates after neglecting a transitional time period (depending on initial data) implies the existence of global attractor for the Boussinesq system at large Prandtl number (54).

This completes our uniform $H^{1}$ estimates.

Next, we estimate the time derivatives which are needed in order to view the Boussinesq system as a perturbation of the infinite Prandtl number model.

We first observe that, according to the perturbative temperature equation (18), and the uniform estimates (68), (61), (40), (45),

$$
\begin{aligned}
\left|\frac{\partial \theta}{\partial t}\right|_{H^{-1}} & \leq|\Delta \theta|_{H^{-1}}+|\mathbf{u} \cdot \nabla \theta|_{H^{-1}}+\left|u_{3}\right|_{H^{-1}} \\
& \leq|\nabla \theta|_{L^{2}}+|\mathbf{u}|_{L^{6}}|\theta|_{L^{3}}+\left|u_{3}\right|_{L^{2}} \\
& \leq|\nabla \theta|_{L^{2}}+c_{6}|\nabla \mathbf{u}|_{L^{2}}|\theta|_{L^{2}}^{\frac{1}{2}}|\nabla \theta|_{L^{2}}^{\frac{1}{2}}+\left|u_{3}\right|_{L^{2}} \\
& \leq c_{7}|\Omega|^{3} R a^{\frac{5}{2}}, \quad \forall t \geq t_{3}+1 .
\end{aligned}
$$


Next, we differentiate the velocity equation (17) in time and deduce

$$
\varepsilon\left(\frac{\partial^{2} \mathbf{u}}{\partial t^{2}}+\left(\frac{\partial \mathbf{u}}{\partial t} \cdot \nabla\right) \mathbf{u}+(\mathbf{u} \cdot \nabla) \frac{\partial \mathbf{u}}{\partial t}\right)+\nabla \frac{\partial p}{\partial t}=\Delta \frac{\partial \mathbf{u}}{\partial t}+R a \mathbf{k} \frac{\partial \theta}{\partial t} .
$$

Multiplying this equation by $\frac{\partial \mathbf{u}}{\partial t}$ and integrating over $\Omega$, we deduce, for $t \geq$ $t_{3}+1$,

$$
\begin{aligned}
& \frac{\varepsilon}{2} \frac{d}{d t}\left|\frac{\partial \mathbf{u}}{\partial t}\right|_{L^{2}}^{2}+\left|\nabla \frac{\partial \mathbf{u}}{\partial t}\right|_{L^{2}}^{2} \\
\leq & R a\left|\frac{\partial \theta}{\partial t}\right|_{H^{-1}}\left|\nabla \frac{\partial \mathbf{u}}{\partial t}\right|_{L^{2}}+\varepsilon|\nabla \mathbf{u}|_{L^{2}}\left|\frac{\partial \mathbf{u}}{\partial t}\right|_{L^{4}}^{2} \\
\leq & R a\left|\frac{\partial \theta}{\partial t}\right|_{H^{-1}}\left|\nabla \frac{\partial \mathbf{u}}{\partial t}\right|_{L^{2}}+c_{8} \varepsilon|\nabla \mathbf{u}|_{L^{2}}\left|\frac{\partial \mathbf{u}}{\partial t}\right|_{L^{2}}^{\frac{1}{2}}\left|\nabla \frac{\partial \mathbf{u}}{\partial t}\right|_{L^{2}}^{\frac{3}{2}} \\
\leq & \frac{1}{4}\left|\nabla \frac{\partial \mathbf{u}}{\partial t}\right|_{L^{2}}^{2}+2 R a^{2}\left|\frac{\partial \theta}{\partial t}\right|_{H^{-1}}^{2}+c_{9} \varepsilon^{4}|\nabla \mathbf{u}|_{L^{2}}^{4}\left|\frac{\partial \mathbf{u}}{\partial t}\right|_{L^{2}}^{2} \\
\leq & \frac{1}{4}\left|\nabla \frac{\partial \mathbf{u}}{\partial t}\right|_{L^{2}}^{2}+2 c_{7}^{2}|\Omega|^{6} R a^{7}+c_{9} 6^{4} \varepsilon^{4}|\Omega|^{4} R a^{4}\left|\frac{\partial \mathbf{u}}{\partial t}\right|_{L^{2}}^{2}
\end{aligned}
$$

where we have utilized (69) and (61).

Therefore,

$$
\varepsilon \frac{d}{d t}\left|\frac{\partial \mathbf{u}}{\partial t}\right|_{L^{2}}^{2}+\left|\nabla \frac{\partial \mathbf{u}}{\partial t}\right|_{L^{2}}^{2} \leq 4 c_{7}^{2}|\Omega|^{6} R a^{7}, t \geq t_{3}+1
$$

provided $\operatorname{Pr}=\frac{1}{\varepsilon}$ is large enough so that

$$
c_{9} 6^{4} \varepsilon^{4}|\Omega|^{4} R a^{4}=c_{9} 6^{4}|\Omega|^{4}\left(\frac{R a}{P r}\right)^{4} \leq \frac{1}{4} .
$$

This is again a large Prandtl number condition as in (54).

Applying Poincaré and Gronwall inequalities, we deduce, for $t \geq t_{*} \geq$ $t_{3}+1$

$$
\begin{aligned}
\left|\frac{\partial \mathbf{u}}{\partial t}(t)\right|_{L^{2}}^{2} \leq & e^{-\frac{t-t^{*}}{\varepsilon}}\left|\frac{\partial \mathbf{u}}{\partial t}\left(t_{*}\right)\right|_{L^{2}}^{2}+4 c_{7}^{2}|\Omega|^{6} R a^{7} \\
\leq & \frac{e^{-\frac{t-t_{*}}{\varepsilon}}}{\varepsilon^{2}}\left(\left|\Delta \mathbf{u}\left(t_{*}\right)\right|_{L^{2}}+R a\left|\theta\left(t_{*}\right)\right|+\varepsilon\left|\left(\mathbf{u}\left(t_{*}\right) \cdot \nabla\right) \mathbf{u}\left(t_{*}\right)\right|_{L^{2}}\right)^{2} \\
& +4 c_{7}^{2}|\Omega|^{6} R a^{7}
\end{aligned}
$$


Thanks to (46), (61) and (54), we have

$$
\begin{aligned}
\varepsilon \frac{d}{d t}|\nabla \mathbf{u}|_{L^{2}}^{2}+|A \mathbf{u}|_{L^{2}}^{2} & \leq 18|\Omega|^{2} R a^{2}+128 c_{2}^{4} \varepsilon^{4}|\nabla \mathbf{u}|_{L^{2}}^{6} \\
& \leq 18|\Omega|^{2} R a^{2}+128 c_{2}^{4} \varepsilon^{4} 6^{6}|\Omega|^{6} R a^{6} \\
& \leq 36|\Omega|^{2} R a^{2}, \quad \forall t \geq t_{3} .
\end{aligned}
$$

This implies, for $t>t_{3}+1$,

$$
\begin{aligned}
\frac{1}{t-t_{3}-1} \int_{t_{3}+1}^{t}|A \mathbf{u}(s)|_{L^{2}}^{2} d s & \leq 36|\Omega|^{2} R a^{2}+\varepsilon\left|\nabla \mathbf{u}\left(t_{3}+1\right)\right|_{L^{2}}^{2} \\
& \leq 72|\Omega|^{2} R a^{2}
\end{aligned}
$$

where we have applied the $H^{1}$ uniform estimate for the velocity (61).

Hence there exists $t^{*} \in\left[t_{3}+1, t_{3}+2\right]$, such that

$$
\left|A \mathbf{u}\left(t^{*}\right)\right|_{L^{2}}^{2} \leq 72|\Omega|^{2} R a^{2}
$$

and thus by elliptic regularity,

$$
\left|\Delta \mathbf{u}\left(t^{*}\right)\right|_{L^{2}}^{2} \leq c_{10}|\Omega|^{2} R a^{2},
$$

which further implies

$$
\begin{aligned}
\left|\left(\mathbf{u}\left(t^{*}\right) \cdot \nabla\right) \mathbf{u}\left(t^{*}\right)\right|_{L^{2}} & \leq c_{11}\left|\nabla \mathbf{u}\left(t^{*}\right)\right|_{L^{2}}^{\frac{3}{2}}\left|\Delta \mathbf{u}\left(t^{*}\right)\right|_{L^{2}}^{\frac{1}{2}} \\
& \leq c_{12}|\Omega|^{2} R a^{2}
\end{aligned}
$$

Combining this estimate with (74), (68), (78) and (54), we have,

$$
\begin{aligned}
\left|\frac{\partial \mathbf{u}}{\partial t}(t)\right|_{L^{2}}^{2} & \leq \frac{c_{13}}{\varepsilon^{2}} e^{-\frac{t-t_{3}-2}{\varepsilon}}\left(|\Omega|^{2} R a^{2}+\varepsilon^{2}|\Omega|^{4} R a^{4}\right)+4 c_{7}^{2}|\Omega|^{6} R a^{7} \\
& \leq c_{14}|\Omega|^{6} R a^{7}, \quad \forall t \geq t_{3}+3
\end{aligned}
$$

since $\frac{1}{\varepsilon} e^{-\frac{1}{\varepsilon}} \leq 4 e^{-2}$.

Next, we differentiate the temperature equation (18) in time and we deduce

$$
\frac{\partial^{2} \theta}{\partial t^{2}}+\mathbf{u} \cdot \nabla \frac{\partial \theta}{\partial t}+\frac{\partial \mathbf{u}}{\partial t} \cdot \nabla \theta-\frac{\partial u_{3}}{\partial t}=\Delta \frac{\partial \theta}{\partial t} .
$$

Multiplying this equation by $\frac{\partial \theta}{\partial t}$, integrating over $\Omega$, we have

$$
\begin{aligned}
\frac{1}{2} \frac{d}{d t}\left|\frac{\partial \theta}{\partial t}\right|_{L^{2}}^{2}+\left|\nabla \frac{\partial \theta}{\partial t}\right|_{L^{2}}^{2} & \leq\left|\frac{\partial u_{3}}{\partial t}\right|_{L^{2}}\left|\frac{\partial \theta}{\partial t}\right|_{L^{2}}+\left|\frac{\partial \mathbf{u}}{\partial t}\right|_{L^{2}}|\nabla \theta|_{L^{6}}\left|\frac{\partial \theta}{\partial t}\right|_{L^{3}} \\
& \leq \frac{1}{2}\left|\nabla \frac{\partial \theta}{\partial t}\right|_{L^{2}}^{2}+\left|\frac{\partial u_{3}}{\partial t}\right|_{L^{2}}^{2}+c_{15}\left|\frac{\partial \mathbf{u}}{\partial t}\right|_{L^{2}}^{2}|\Delta \theta|_{L^{2}}^{2}
\end{aligned}
$$


Combining this with (80) and Poincaré inequality, we have, for $t \geq t_{3}+3$,

$$
\frac{d}{d t}\left|\frac{\partial \theta}{\partial t}\right|_{L^{2}}^{2}+\left|\frac{\partial \theta}{\partial t}\right|_{L^{2}}^{2} \leq c_{16}|\Omega|^{6} R a^{7}\left(1+|\Delta \theta|_{L^{2}}^{2}\right) .
$$

On the other hand, thanks to (66) and (68), we have

$$
\frac{d}{d t}|\nabla \theta|_{L^{2}}^{2}+|\nabla \theta|_{L^{2}}^{2}+|\Delta \theta|_{L^{2}}^{2} \leq c_{17}|\Omega|^{10} R a^{9}, \quad \forall t \geq t_{3}+1
$$

This implies, together with (68) and a Gronwall type argument,

$$
\begin{aligned}
e^{-t} \int_{t_{3}+3}^{t} e^{s}|\Delta \theta(s)|_{L^{2}}^{2} d s & \leq e^{-\left(t-t_{3}-3\right)}\left|\nabla \theta\left(t_{3}+3\right)\right|_{L^{2}}^{2}+c_{17}|\Omega|^{10} R a^{9} \\
& \leq c_{18}|\Omega|^{10} R a^{9}
\end{aligned}
$$

and

$$
\begin{aligned}
\int_{t_{3}+3}^{t}|\Delta \theta(s)|_{L^{2}}^{2} d s & \leq c_{17}|\Omega|^{10} R a^{9}\left(t-t_{3}-3\right)+\left|\nabla \theta\left(t_{3}+3\right)\right|_{L^{2}}^{2} \\
& \leq c_{19}|\Omega|^{10} R a^{9}\left(t-t_{3}-2\right) .
\end{aligned}
$$

Therefore, there exists $t^{*} \in\left[t_{3}+3, t_{3}+4\right]$, such that

$$
\left|\Delta \theta\left(t^{*}\right)\right|^{2} \leq 2 c_{19}|\Omega|^{10} R a^{9} .
$$

Hence

$$
\begin{aligned}
& \left|\frac{\partial \theta}{\partial t}\left(t^{*}\right)\right|_{L^{2}} \\
\leq & \left|\Delta \theta\left(t^{*}\right)\right|_{L^{2}}+\left|u_{3}\left(t^{*}\right)\right|_{L^{2}}+\left|\mathbf{u}\left(t^{*}\right)\right|_{L^{6}}\left|\nabla \theta\left(t^{*}\right)\right|_{L^{3}} \\
\leq & \left|\Delta \theta\left(t^{*}\right)\right|_{L^{2}}+\left|u_{3}\left(t^{*}\right)\right|_{L^{2}}+c_{20}\left|\nabla \mathbf{u}\left(t^{*}\right)\right|_{L^{2}}\left|\nabla \theta\left(t^{*}\right)\right|_{L^{2}}^{\frac{1}{2}}\left|\Delta \theta\left(t^{*}\right)\right|_{L^{2}}^{\frac{1}{2}} \\
\leq & c_{21}|\Omega|^{5} R a^{\frac{5}{4}}
\end{aligned}
$$

We now apply Gronwall inequality to (83) and utilize (85) and (88), and we deduce

$$
\begin{aligned}
\left|\frac{\partial \theta}{\partial t}(t)\right|_{L^{2}}^{2} & \leq e^{-\left(t-t^{*}\right)}\left|\frac{\partial \theta}{\partial t}\left(t^{*}\right)\right|_{L^{2}}^{2}+c_{22}|\Omega|^{16} R a^{16} \\
& \leq c_{23}|\Omega|^{16} R a^{16}, \quad \forall t \geq t_{3}+4
\end{aligned}
$$

Thus we have completed the uniform $L^{2}$ estimates of the time derivatives. 
We are left with the uniform $H^{2}$ estimates.

For this purpose, we multiply the velocity equation (17) by $A \mathbf{u}$ and integrate over $\Omega$ and deduce

$$
\begin{aligned}
& |A \mathbf{u}(t)|_{L^{2}}^{2} \\
\leq & R a|A \mathbf{u}(t)|_{L^{2}}|\theta(t)|_{L^{2}}+\varepsilon\left|\frac{\partial \mathbf{u}}{\partial t}(t)\right|_{L^{2}}|A \mathbf{u}(t)|_{L^{2}}+\varepsilon|\mathbf{u}(t)|_{L^{\infty}}|\nabla \mathbf{u}(t)|_{L^{2}}|A \mathbf{u}(t)|_{L^{2}} \\
\leq & R a|A \mathbf{u}(t)|_{L^{2}}|\theta(t)|_{L^{2}}+\varepsilon\left|\frac{\partial \mathbf{u}}{\partial t}(t)\right|_{L^{2}}|A \mathbf{u}(t)|_{L^{2}}+c_{24} \varepsilon|\nabla \mathbf{u}(t)|_{L^{2}}^{\frac{3}{2}}|A \mathbf{u}(t)|_{L^{2}}^{\frac{3}{2}} \\
\leq & \frac{1}{2}|A \mathbf{u}(t)|_{L^{2}}^{2}+4 R a^{2}|\theta(t)|_{L^{2}}^{2}+4 \varepsilon^{2}\left|\frac{\partial \mathbf{u}}{\partial t}(t)\right|_{L^{2}}^{2}+c_{25} \varepsilon^{4}|\nabla \mathbf{u}(t)|_{L^{2}}^{6}
\end{aligned}
$$

Hence, for $t \geq t_{3}+3$, we have

$$
\begin{aligned}
|A \mathbf{u}(t)|_{L^{2}}^{2} & \leq 8 R a^{8}|\theta(t)|_{L^{2}}^{2}+8 \varepsilon^{2}\left|\frac{\partial \mathbf{u}}{\partial t}(t)\right|_{L^{2}}^{2}+2 c_{25} \varepsilon^{4}|\nabla \mathbf{u}(t)|_{L^{2}}^{6} \\
& \leq 72 R a^{2}|\Omega|^{2}+8 \varepsilon^{2} c_{14}|\Omega|^{6} R a^{7}+c_{26} \varepsilon^{4}|\Omega|^{6} R a^{6} \\
& \leq c_{27}|\Omega|^{4} R a^{5}
\end{aligned}
$$

where we have used the large Prandtl number assumption (54) and the large Rayleigh number and aspect ratio assumption.

Elliptic regularity then implies,

$$
|\mathbf{u}(t)|_{H^{2}} \leq c_{28}|\Omega|^{2} R a^{\frac{5}{2}}, \quad \forall t \geq t_{3}+3 . .
$$

As for the $H^{2}$ estimate for the temperature field, we have

$$
\begin{aligned}
|\Delta \theta(t)|_{L^{2}} & \leq\left|\frac{\partial \theta}{\partial t}(t)\right|_{L^{2}}+\left|u_{3}(t)\right|_{L^{2}}+|\mathbf{u}(t)|_{L^{\infty}}|\nabla \theta(t)|_{L^{2}} \\
& \leq\left|\frac{\partial \theta}{\partial t}(t)\right|_{L^{2}}+\left|u_{3}(t)\right|_{L^{2}}+c_{29}|\nabla \mathbf{u}(t)|_{L^{2}}^{\frac{1}{2}}|\mathbf{u}(t)|_{H^{2}}^{\frac{1}{2}}|\nabla \theta(t)|_{L^{2}} \\
& \leq c_{30}|\Omega|^{8} R a^{8}
\end{aligned}
$$

where we have applied the uniform estimates (89), (45), (61), (92), and (68).

This completes our uniform estimates in $H^{2}$.

To summarize, we have the following

Lemma 1 (Uniform a priori estimates) Let Ra be an arbitrary large but fixed Rayleigh number. Suppose the Prandtl number Pr is large enough so that the conditions (54, 73) are satisfied. Then for any given suitable 
weak solutions $(\mathbf{u}(t), \theta(t))$ of the Boussinesq system, there exists a time $t_{3}=$ $t_{3}\left(\left|\mathbf{u}_{0}\right|_{L^{2}},\left|T_{0}\right|_{L^{2}},|\Omega|\right)$ given explicitly in (58), and a constant $c_{31}$ independent of $\mathrm{Pr}, \mathrm{Ra}$ such that the following hold when $t \geq t_{3}+4$

$$
\begin{aligned}
|\mathbf{u}(t)|_{H^{2}} & \leq c_{31}|\Omega|^{2} R a^{\frac{5}{2}}, \\
|\theta(t)|_{H^{2}} & \leq c_{31}|\Omega|^{8} R a^{8}, \\
\left|\frac{\partial \mathbf{u}}{\partial t}(t)\right|_{L^{2}} & \leq c_{31}|\Omega|^{3} R a^{\frac{7}{2}}, \\
\left|\frac{\partial \theta}{\partial t}(t)\right|_{L^{2}} & \leq c_{31}|\Omega|^{8} R a^{8} .
\end{aligned}
$$

In particular, solutions on any of the global attractor must satisfy these estimates.

\section{Convergence of the Global Attractors}

We now show the convergence of the global attractors of the Boussinesq system to that of the infinite Prandtl number model as the Prandtl number approaches infinity.

As we mentioned earlier, the natural phase space for the Boussinesq system and the infinite Prandtl number model are different with the Boussinesq system requiring both the velocity and the temperature field while the infinite Prandtl number model has only the temperature field (or velocity field).

There are two natural approaches to handle this discrepancy in phase space. We either project the phase space of the Boussinesq system down to the temperature filed only, or we lift the phase space for the infinite Prandtl number model to the product space of velocity and temperature. We will see that the comparison of global attractors after projection is relatively easy, and is similar to the upper semi-continuity of global attractors for dynamical systems (see for instance $[18,36,33])$. The comparison of global attractors after lifting the phase space of the infinite Prandtl number model is a little bit more involved. Here we view the Boussinesq system as a small perturbation of the infinite Prandtl number model with the help of the a priori estimates that we derived in section 3. The convergence of the global attractors follows from appropriate a priori estimates (uniform in Prandtl number) on the material derivative of the velocity field after the initial layer.

It is our belief that the techniques developed here can be applied to more general dynamical systems with two explicitly separated time scales 
(see section 4 as well). Therefore, we formulate our result in a more general fashion and view the case of convection at large Prandtl number as a special application.

Theorem 1 (Convergence of Global Attractors) Consider a generalized dynamical system on $X_{1} \times X_{2}$ with two explicitly separated time scales

$$
\begin{aligned}
\varepsilon\left(\frac{d x_{1}}{d t}+g\left(x_{1}, x_{2}\right)\right) & =f_{1}\left(x_{1}, x_{2}\right), x_{1}(0)=x_{10}, \\
\frac{d x_{2}}{d t} & =f_{2}\left(x_{1}, x_{2}\right), x_{2}(0)=x_{20},
\end{aligned}
$$

where $X_{1}, X_{2}$ are two Banach spaces.

Let

$$
\begin{aligned}
0 & =f_{1}\left(x_{1}^{0}, x_{2}^{0}\right), \\
\frac{d x_{2}^{0}}{d t} & =f_{2}\left(x_{1}^{0}, x_{2}^{0}\right), x_{2}(0)=x_{20}
\end{aligned}
$$

be the limit system.

We postulate the following assumptions:

$H 1$ (uniform dissipativity of the perturbed system) The two-time-scale system $(98,99)$ possesses a global attractor $\mathcal{A}_{\varepsilon}$ for all small positive $\varepsilon$. We also assume that the global attractors are regular and uniformly bounded in the sense that there exist Banach spaces $Y_{j}, j=1,2$ which are continuously imbedded in the $X_{j}, j=1,2$ respectively, and there exists a constant $R_{0}$ such that

$$
\left\|x_{1}\right\|_{Y_{1}}+\left\|x_{2}\right\|_{Y_{2}} \leq R_{0}, \forall\left(x_{1}, x_{2}\right) \in \mathcal{A}_{\varepsilon}, \forall \varepsilon
$$

H2 (dissipativity of the limit system) The limit system is wellposed and possesses a global attractor $\mathcal{A}_{0}$ in $X_{2}$.

H3 (convergence of the slow variable) The slow variable of the solutions of the two time scale system converge uniformly on bounded sets in $Y_{1} \times Y_{2}$ to that of the limit system after neglecting a transitional time period, i.e., for any $R>0$, there exists a $t_{0}>0$ such that for any $t>t_{0}$,

$$
\lim _{\varepsilon \rightarrow 0} \sup _{\left\|x_{10}\right\|_{Y_{1}}+\left\|x_{20}\right\|_{Y_{2}} \leq R}\left\|x_{2}(t)-x_{2}^{0}(t)\right\|_{X_{2}}=0, \forall t \geq t_{0} .
$$


Then the global attractors $\mathcal{A}_{\varepsilon}$ of the two time scale system converge to $\mathcal{A}_{0}$ after projection, i.e.

$$
\lim _{\varepsilon \rightarrow 0} \operatorname{dist}_{X_{2}}\left(\mathcal{P}_{2} \mathcal{A}_{\varepsilon}, \mathcal{A}_{0}\right)=0,
$$

where $\mathcal{P}_{2}$ is the projection from $X_{1} \times X_{2}$ to $X_{2}$ defined as

$$
\mathcal{P}_{2}\left(x_{1}, x_{2}\right)=x_{2}
$$

Furthermore, if we assume

$\mathrm{H}_{4}$ (smallness of the perturbation) The two time scale problem $(98,99)$ is a uniformly small perturbation of the limit problem $(100,101)$ when confined to the global attractors, i.e.,

$$
\lim _{\varepsilon \rightarrow 0} \sup _{\left(x_{1}, x_{2}\right) \in \mathcal{A}_{\varepsilon}}\left\|\varepsilon\left(\frac{d x_{1}}{d t}+g\left(x_{1}, x_{2}\right)\right)\right\|_{X_{1}}=0 .
$$

H5 (continuity of the slave relation) The first equation in the limit system (100) can be solved continuously for $x_{1}^{0}$ with given $x_{2}^{0}$ and a nontrivial left hand side, i.e., there exists a continuous function $F_{1}: X_{2} \times X_{1} \rightarrow$ $X_{1}$ such that

$$
y=f_{1}\left(F_{1}\left(x_{2}, y\right), x_{2}\right) .
$$

Moreover, we assume $F_{1}$ is uniformly continuous for $y=0$ and $x_{2}$ in bounded sets in $Y_{2}$.

Then the attractors $\mathcal{A}_{\varepsilon}$ of the two time scale system converge to $\mathcal{A}_{0}$ after lift, i.e.

$$
\lim _{\varepsilon \rightarrow 0} \operatorname{dist}_{X_{1} \times X_{2}}\left(\mathcal{A}_{\varepsilon}, \mathcal{L A}_{0}\right)=0
$$

where $\mathcal{L}$ is the lift from $X_{2}$ to $X_{1} \times X_{2}$ defined by

$$
\mathcal{L}\left(x_{2}\right)=\left(F_{1}\left(x_{2}, 0\right), x_{2}\right) .
$$

\section{Proof of the theorem}

We borrow ideas from the proof of upper semi-continuity of global attractors for dissipative dynamical systems [18, 36, 33].

Recall that the Hausdorff semi-distance between two sets $A_{1}, A_{2}$ in a Banach space is defined as

$$
\operatorname{dist}_{X}\left(A_{1}, A_{2}\right)=\sup _{x_{1} \in A_{1}} \inf _{x_{2} \in A_{2}}\left\|x_{1}-x_{2}\right\|_{X}
$$


We first prove the convergence in the projected sense, i.e., (104).

Let $\delta>0$ be fixed. Since the limit system possesses a global attractor $\mathcal{A}_{0}$ (H2), and since a bounded ball in $Y_{2}$ is bounded in $X_{2}$ by the continuous imbedding (H1), and since the global attractor attracts all bounded set in the phase space, there exists a $T=T(\delta)>0$ such that

$$
\operatorname{dist}_{X_{2}}\left(S^{0}(t) B_{R_{0}}\left(Y_{2}\right), \mathcal{A}_{0}\right) \leq \frac{\delta}{3}, \forall t \geq T
$$

where $S^{0}(t)$ denotes the solution semi-group associated with the limit system $(100,101)$ and $B_{R_{0}}\left(Y_{2}\right)$ denotes the ball in $Y_{2}$ with radius $R_{0}$ centered at the origin.

On the other hand, utilizing $\mathrm{H} 3$ with $R=R_{0}$, we see that there exists an $\varepsilon(\delta)$ such that

$$
\left.\sup _{\left(x_{10}, x_{20}\right) \in B_{R_{0}}\left(Y_{1} \times Y_{2}\right)}\left\|x_{2}(T)-x_{2}^{0}(T)\right\|_{X_{2}} \leq \frac{\delta}{2}, \forall \varepsilon \leq \varepsilon(\delta) .\right)
$$

Now for $\left(y_{1}, y_{2}\right) \in \mathcal{A}_{\varepsilon}$ with $\varepsilon \leq \varepsilon(\delta)$, there exists $\left(x_{10}, x_{20}\right) \in \mathcal{A}_{\varepsilon} \subset$ $B_{R_{0}}\left(Y_{1} \times Y_{2}\right)$ so that

$$
\left(y_{1}, y_{2}\right)=\left(x_{1}(T), x_{2}(T)\right)
$$

since $\mathcal{A}_{\varepsilon}$ is invariant.

Therefore, thanks to (112),

$$
\left\|y_{2}-x_{2}^{0}(T)\right\|_{X_{2}}=\left\|x_{2}(T)-x_{2}^{0}(T)\right\|_{X_{2}} \leq \frac{\delta}{2}
$$

On the other hand, since $x_{2}^{0}(T)=S^{0}(T) x_{20} \in S^{0}(T) B_{R_{0}}\left(Y_{2}\right)$, there exists a $x_{2 \infty}^{0} \in \mathcal{A}_{0}$ such that

$$
\left\|x_{2}^{0}(T)-x_{2 \infty}^{0}\right\|_{X_{2}} \leq \frac{\delta}{2}
$$

by the attracting property of $\mathcal{A}_{0}(111)$.

Hence we deduce, by triangle inequality,

$$
\left\|y_{2}-x_{2 \infty}^{0}\right\|_{X_{2}} \leq \delta
$$

This further implies

$$
\operatorname{dist}_{X_{2}}\left(y_{2}, \mathcal{A}_{0}\right) \leq \delta .
$$

Since $y_{2}$ is an arbitrary element in $\mathcal{P}_{2} \mathcal{A}_{\varepsilon}, \varepsilon \leq \varepsilon(\delta)$, we have

$$
\operatorname{dist}_{X_{2}}\left(\mathcal{P}_{2} \mathcal{A}_{\varepsilon}, \mathcal{A}_{0}\right) \leq \delta, \quad \forall \varepsilon \leq \varepsilon(\delta) .
$$


This ends the proof of the convergence of the global attractors in the projected sense, i.e., (104).

Next we discuss convergence in the lifted sense as defined in (108).

Thanks to H5, we can rewrite the fast equation (98) as

$$
x_{1}=F_{1}\left(x_{2}, \varepsilon\left(\frac{d x_{1}}{d t}+g\left(x_{1}, x_{2}\right)\right)\right) .
$$

We also notice that for any fixed $\delta>0$, there exists an $\eta=\eta(\delta)>0$ such that

$$
\left\|F_{1}\left(x_{2}, y\right)-F_{1}\left(x_{2}^{0}, 0\right)\right\|_{X_{1}} \leq \frac{\delta}{2}
$$

provided that

$$
\left\|x_{2}-x_{2}^{0}\right\|_{X_{2}}+\|y\|_{X_{1}} \leq \eta
$$

by the uniform continuity of $F_{1}$ (H5).

Thanks to H4, we have, for some $\varepsilon_{1}=\varepsilon_{1}(\delta)$,

$$
\sup _{\left(x_{1}, x_{2}\right) \in \mathcal{A}_{\varepsilon}}\left\|\varepsilon\left(\frac{d x_{1}}{d t}+g\left(x_{1}, x_{2}\right)\right)\right\|_{X_{1}} \leq \frac{\eta}{2}, \forall \varepsilon \leq \varepsilon_{1} .
$$

We also have, thanks to the first part of the theorem, there exists an $\varepsilon_{2}=\varepsilon_{2}(\delta)$, such that

$$
\operatorname{dist}_{X_{2}}\left(\mathcal{P}_{2} \mathcal{A}_{\varepsilon}, \mathcal{A}_{0}\right) \leq \min \left(\frac{\delta}{3}, \frac{\eta}{3}\right), \forall \varepsilon \leq \varepsilon_{2} .
$$

Therefore, for any given $\left(x_{1}, x_{2}\right) \in \mathcal{A}_{\varepsilon}, \varepsilon \leq \min \left(\varepsilon_{1}, \varepsilon_{2}\right)$, there exists an $x_{2}^{0} \in$ $\mathcal{A}_{0}$ such that

$$
\left\|x_{2}-x_{2}^{0}\right\|_{X_{2}} \leq \min \left(\frac{\delta}{2}, \frac{\eta}{2}\right)
$$

Consequently, for $\varepsilon \leq \min \left(\varepsilon_{1}, \varepsilon_{2}\right)$, we have

$$
\left\|x_{2}-x_{2}^{0}\right\|_{X_{2}}+\left\|\varepsilon\left(\frac{d x_{1}}{d t}+g\left(x_{1}, x_{2}\right)\right)\right\|_{X_{1}} \leq \eta
$$

and hence

$$
\begin{aligned}
& \left\|x_{1}-x_{1}^{0}\right\|_{X_{1}}+\left\|x_{2}-x_{2}^{0}\right\|_{X_{2}} \\
= & \left\|F_{1}\left(x_{2}, \varepsilon\left(\frac{d x_{1}}{d t}+g\left(x_{1}, x_{2}\right)\right)\right)-F_{1}\left(x_{2}^{0}, 0\right)\right\|_{X_{1}}+\left\|x_{2}-x_{2}^{0}\right\|_{X_{2}} \\
\leq & \delta .
\end{aligned}
$$


Henceforth

$$
\operatorname{dist}_{X_{1} \times X_{2}}\left(\left(x_{1}, x_{2}\right), \mathcal{L} \mathcal{A}_{0}\right) \leq \delta .
$$

This end the proof of the theorem.

An immediate consequence of the theorem is its application to the Boussinesq system for convection at large Prandtl number. We have

Corollary 1 (Application to large Prandtl number convection) For any large but fixed Rayleigh number $R a$, the global attractors $\mathcal{A}_{\varepsilon}$ of the Boussinesq system for convection with Prandtl number $\operatorname{Pr}=\frac{1}{\varepsilon}$ converge to the attractor $\mathcal{A}_{0}$ of the infinite Prandtl number model for convection in both the projected sense of (104) and the lifted sense of (108) as $\varepsilon$ approaches zero.

Proof of the Corollary We need to verify the assumptions in the theorem.

We first identify

$$
\begin{aligned}
X_{1} & =\left\{\mathbf{u} \in \mathbf{L}^{2}\left|u_{3}\right|_{z=0,1}=0, \nabla \cdot \mathbf{u}=0, \text { periodic in } x \text { and } y\right\} \\
X_{2} & =L^{2} \\
Y_{1} & =\left\{\mathbf{u} \in \mathbf{H}^{2}|\mathbf{u}|_{z=0,1}=0, \nabla \cdot \mathbf{u}=0, \text { periodic in } x \text { and } y\right\}, \\
Y_{2} & =\left\{\theta \in H^{2}|\theta|_{z=0,1}=0, \text { periodic in } x \text { and } y\right\} .
\end{aligned}
$$

It is then obvious that the Hilbert spaces $Y_{j}, j=1,2$ are continuously imbedded in the Hilbert spaces $X_{j}, j=1,2$ respectively.

Hypothesis 1 is clear thanks to Lemma 1 and [42] where we have shown the existence of global attractors for the Boussinesq system for convection.

Hypothesis 2 is evident with our infinite Prandtl number model.

Hypothesis 3 is verified thanks to Theorem 2 of [41] where we proved the convergence of solutions of the Boussinesq system to that of the infinite Prandtl number model on finite time interval after neglecting a transitional time period and with initial data in $Y_{1} \times Y_{2}$. The uniformity of convergence is clear since the constants depend on the $Y_{1} \times Y_{2}$ norm only.

As for Hypothesis 4, we have

$$
\begin{aligned}
\left|\varepsilon\left(\frac{\partial \mathbf{u}}{\partial t}+(\mathbf{u} \cdot \nabla) \mathbf{u}\right)\right|_{L^{2}} & \leq \varepsilon\left(\left|\frac{\partial \mathbf{u}}{\partial t}\right|_{L^{2}}+|\mathbf{u}|_{L^{\infty}}|\nabla \mathbf{u}|_{L^{2}}\right) \\
& \leq \varepsilon\left(\left|\frac{\partial \mathbf{u}}{\partial t}\right|_{L^{2}}+c_{32}|\mathbf{u}|_{H^{2}}|\nabla \mathbf{u}|_{L^{2}}\right) \\
& \rightarrow 0
\end{aligned}
$$


according to lemma 1. Thus Hypothesis 4 is verified.

In terms of Hypothesis 5, we have, for $(\mathbf{v}, \theta) \in Y_{1} \times Y_{2}$,

$$
F_{1}(\theta, \mathbf{v})=A^{-1}(R a P(\mathbf{k} \theta)-\mathbf{v})
$$

where $A$ is the Stokes operator and $P$ is the Leray-Hopf projection from $\mathbf{L}^{2}$ to $X_{1}[8,11,17,37]$. It is then clear that Hypothesis 5 is satisfied.

This ends the proof of the corollary.

Remark: It is worthwhile to reiterate that the global attractors to the Boussinesq system for convection and the infinite Prandtl number model for convection are non-trivial. It is easy to see that the pure conduction state belongs to each of the global attractors. It is well-known that the pure conduction state becomes unstable at high enough Rayleigh number and Bénard cells emerge $[32,43,26]$. Hence the attractors must be non-trivial. Indeed, numerical results indicate convection at large Prandtl number could be very complicated with thermal plumes emerge at seemingly random time and at random location [39].

\section{Comments}

In this manuscript we have demonstrated that the global attractors of the Boussinesq system (which exist at large Prandtl number [42]) converge to that of the infinite Prandtl number model for convection. This complements our earlier result on the convergence of weak solutions to the Boussinesq system to that of the infinite Prandtl number model on finite interval modulo an initial transitional layer [41]. These two results provide us with confidence of using the infinite Prandtl number model as a simplified model for convection at large Prandtl number, both on short time and on long time since the global attractors embody all long time behavior.

On the other hand, convergence in the symmetric Hausdorff distance sense, i.e., replace $\operatorname{dist}\left(A_{1}, A_{2}\right)$ by $\operatorname{dist}\left(A_{1}, A_{2}\right)+\operatorname{dist}\left(A_{2}, A_{1}\right)$ for two sets in a Banach or metric space, usually requires some hyperbolicity $[1,36,33]$ and may be much harder or even invalid as we can see from simple bifurcation examples such as

$$
\frac{d u}{d t}=u\left(-1+2 u^{2}-u^{4}-\epsilon\right)
$$

where the global attractor for the case of $\varepsilon>0$ is the origin only and the global attractor for the case of $\varepsilon \leq 0$ includes the interval $[-1,1]$. 
There are two immediate questions that come naturally. First, are there any other good measures of the (long time) validity of the infinite Prandtl number model? If yes, what can we say about the the validity under these measures? Second, is the result here special or could be applied to more general (generalized) dynamical systems with two explicitly separated time scales?

Regarding the first question, there are many other measures of (long time) validity of the infinite Prandtl number model. For instance, it is easy to show that the Hausdorff/fractal dimension of the global attractor to the Boussinesq system at large Prandtl number and that of the infinite Prandtl number are finite. Thus we may ask if the dimension of the global attractors to the Boussinesq system converge to that of the infinite Prandtl number model. This could be very hard since we are usually only able to estimate upper bounds for the dimension of attractors. We can then ask whether there is a uniform in (large) Prandtl number bound on the dimension of attractors for the Boussinesq system. The direct application of the Constantin-FoiasTemam version of the Kaplan-Yorke formula ([36]) seems not working and may need revision due to the two time scales. The theorem that we have in section 3 supports an affirmative answer to the question of existence of uniform bounds on the global attractor. However, it is not conclusive since two very close sets could have very different fractal dimensions due to local oscillations. Therefore, it also makes sense to discuss convergence of trajectory and attractors in space with more regularity (which measures oscillation). An alternative approach is to use the crude estimates using squeezing properties $[23,36,35]$. One may also consider bounds on the number of determining modes, nodes, volumes etc (see for instance [17] among others).

Another two objects related to long time behavior are inertial manifold and exponential attractors $([36,14])$. It is easy to check that the infinite Prandtl number model for convection possesses an exponential attractor which is positively invariant and attracts all orbits with exponential rate. We can ask if the Boussinesq system possesses exponential attractor at large Prandtl number and whether the exponential attractors, if they exist, converge in some sense to one of the exponential attractors of the infinite Prandtl number model. See [16] for such a convergence result for a singularly perturbed wave equation, [19] for a general result, and see [14] for more on exponential attractors. Indeed, one may even hopeful for continuity of exponential attractors since these objects are more stable $[14,30]$. The question regarding inertial manifold (a finite dimensional manifold which is positively 
invariant under the dynamics and attracts all orbits with exponential rate) is much harder. Even the existence of inertial manifold for the simplified infinite Prandtl number model is unknown.

A related result is a connection to the Landau-Lifschitz theory on degrees of freedom for turbulent flows. With fixed Rayleigh and Prandtl number, the intensity of the turbulence is fixed and thus it is expected that the degrees of freedom of the system scale linearly in each of the horizontal length $\left(L_{x}\right.$ and $L_{y}$ ) according to the Landau-Lifschitz theory (see for instance [11, 17]). Utilizing techniques that we developed earlier for shear flows in elongated channels ([12]) we can obtain upper bounds on the dimension of attractors for the Boussinesq system and the infinite Prandtl number model that scale linearly in each of the horizontal length $\left(L_{x}\right.$ and $\left.L_{y}\right)$. We leave the detail to the interested reader. Such a bound is optimal in terms of dependence on $L_{x}$ and $L_{y}$ since one can show that at a fixed value of Ra the number of linearly unstable modes around the conduction state is proportional to the "density of states" which is proportional to $L_{x} * L_{y}$ (C.R. Doering, private communication). Similar optimal bounds in the case of free-slip boundary condition on top and bottom and with restriction to the two-dimensional case or certain functional invariant sets in the three-dimensional case is known [29],[31].

As we discussed earlier, statistical behavior is probably more important and realistic for systems like the Boussinesq system where we expect turbulent/chaotic behavior. Thus a more important criterion for the validity of the infinite Prandtl number model for convection is if the statistical properties for the Boussinesq system are close to the corresponding statistical properties of the infinite Prandtl number model. A prominent statistical quantity in convection is the averaged heat transfer in the vertical direction which can be characterized via the time averaged Nusselt number ([4], [39], [7]). In the case of infinite Prandtl number model, an upper bound on the Nusselt number which agrees with physical scaling (modulo a logarithmic term) has been derived by Constantin and Doering [7]. It is then interesting to see if we can derive an upper bound on the Nusselt number for the Boussinesq system which agrees with Constantin-Doering result in the sense the upper bound should be the Constantin-Doering bound for infinite Prandtl number convection plus a correction term that vanishes as the Prandtl number approaches infinity. This and a few other issues are currently under investigation [13].

In terms of the second question, we consider the Rayleigh-Bénard convection problem at large Prandtl number as a special case of more general 
physical systems with two explicitly separated time scales. In general we should not expect such kind results to be true all the time. For instance, problems with fast oscillation on the fast time scale cannot be expected to converge in strong sense as is clear from the following example

$$
\begin{aligned}
\varepsilon \frac{d x}{d t} & =-y-z, \\
\varepsilon \frac{d y}{d t} & =x-z, \\
\frac{d z}{d t} & =-z .
\end{aligned}
$$

We see that the limit problem $(\varepsilon=0)$ has trivial dynamics (converge to the origin) while we have persistent oscillation for positive $\varepsilon$. We have neither convergence in terms of individual trajectory, nor in terms of long time behavior. This example can be modified into a dissipative one easily via applying a filter in space. Although strong convergence is not possible in this oscillatory situation, it may be still be possible to discuss convergence in weak sense (see for instance [27]). Oscillation could occur spontaneously via Hopf bifurcation. For instance, the two-dimensional Navier-Stokes equation under generalized Kolmogorov forcing may experience Hopf bifurcation [6]. Therefore it may be hopeful to deal with the case with explicit fast oscillation, it may still be very hard to handle general situation with oscillation generated by the nonlinear mechanism.

If the fast dynamics is not oscillatory, and the limit system is regular enough, we expect similar results. For instance, we expect to have similar results (convergence of trajectory modulo a transitional layer and convergence of global attractor) for convection in porous medium at small Darcy-Prandtl number [38]. Convection in porous medium is more regular than standard convection since the nonlinear advection term in the velocity field is missing and the wellposedness of the governing system is known [15], [25]. We also expect to have similar results for certain reaction-diffusion systems with one fast reaction/diffusion time. In this case, the limit system may not be wellposed since the limit elliptic equation in the fast variable may not have unique solution. In this case we employ the notion of generalized dynamical systems [2] and it seems that similar results may follow as well. 


\section{Acknowledgments}

This work is support in part by a grant from the National Science Foundation, a COFRS award from Florida State University and a senior visiting scholar fellowship from Fudan University. The author thanks C.R. Doering and A. Miranville for helpful suggestions and comments.

\section{References}

[1] A.V. Babin and M.I. Vishik, Attractors of evolutionary equations, NorthHolland, Amsterdam, 1992.

[2] J.M. Ball, Continuity properties and global attractors of generalized semiflows and the Navier-Stokes equations. Mechanics: from theory to computation, 447-474, Springer, New York, 2000.

[3] E. Bodenschatz, W. Pesch and G. Ahlers, Recent developments in Rayleigh-Bnard convection. Annual review of fluid mechanics, Vol. 32, 709-778, 2000.

[4] F.H. Busse, Fundamentals of thermal convection. In Mantle Convection: Plate Tectonics and Global Dynamics, ed WR Peltier, pp. 23-95. Montreux: Gordon and Breach, 1989.

[5] S. Chandrasekhar, Hydrodynamic and hydro-magnetic stability. Oxford, Clarendon Press, 1961.

[6] Z.-M. Chen and W.G. Price, Time dependent periodic Navier-Stokes flows on a two-dimensional torus. Comm. Math. Phys. 179 (1996), no. $3,577-597$

[7] P. Constantin and C. R. Doering, Infinite Prandtl number convection, J. Stat. Phys. 94 (1999), no. 1-2, 159-172.

[8] P. Constantin and C. Foias, Navier-Stokes Equations, Chicago University Press.

[9] P. Constantin, C. Hallstrom, V. Poutkaradze, Logarithmic bounds for infinite Prandtl number rotating convection, J. Math. Phys. 42, no. 2, 773-783, 2001. 
[10] C. R. Doering and P. Constantin, On upper bounds for infinite Prandtl number convection with or without rotation, J. Math. Phys. 42, no. 2, 784-795, 2001.

[11] C.R. Doering and J. Gibbons, Applied Analysis of the Navier-Stokes Equations, Cambridge University Press, Cambridge, UK.

[12] C.R. Doering and X. Wang, Attractor dimension estimates for twodimensional shear flows, Physica D, 123 (1998), pp. 206-222.

[13] C.R. Doering and X. Wang, Upper bounds on Rayleigh-Bénard convection at large Prandtl number, in preparation, 2005.

[14] A. Eden, C. Foias, B. Nicolaenko and R. Temam, Exponential attractors for dissipative evolution equations, John-Wiley, New York, 1994.

[15] P.Fabrie, Existence, unicité et comportement asymptoticque de la solutions d'un problem de convection en mileu poreux, Comptes Rendus de l'Academie des Sciences-Série I, 295, 423-425, 1982.

[16] P. Fabrie, C. Galusinski, A. Miranville and S. Zelik, Uniform exponential attractors for a singularly perturbed damped wave equation, DCDS, vol. 10, no. 1\&2, (2004), pp.211-238.

[17] C. Foias, O. Manley, R. Rosa, R. Temam, Navier-Stokes equations and turbulence. Encyclopedia of Mathematics and its Applications, 83. Cambridge University Press, Cambridge, 2001.

[18] J. K. Hale, Asymptotic behavior of dissipative systems, AMS, Rhode Island, 1988.

[19] S. Gatti, M. Grasselli, A. Miranville and V. Pata, A construction of a robust family of exponential attractors, preprint, 2005.

[20] A.V. Getling, Rayleigh-Bénard convection. Structures and dynamics. Advanced Series in Nonlinear Dynamics, 11. World Scientific Publishing Co., Inc., River Edge, NJ, 1998.

[21] S. Grossmann and D. Lohse, Scaling in thermal convection: a unifying theory, J. Fluid Mech., vol. 407, 2000, pp.27-56. 
[22] O.A. Ladyzhenskaya, The Mathematical Theory of Viscous Incompressible Flows,2ed Gordon and Breach, New York, 1969.

[23] O.A. Ladyzhenskaya, Attractors for semigroups and evolution equations, Cambridge ; New York : Cambridge University Press, 1991.

[24] J.L. Lions, Quelques Methodes de Resolution des Provlémes aux Limites non Linéaires, Dunod, Paris, 1969.

[25] H.V. Ly and E.S. Titi, Global Gevrey regularity for the Bénard convection in porous medium with zero Darcy-Prandtl number, Jour. Nonlinear Sci., 9, 333-362, 1999.

[26] T. MA AND S. WANG, Dynamic bifurcation and stability in the Rayleigh-Bénard convection, Comm. Math. Sci. vol.2, No.2, (2004), pp. 159-183.

[27] A.J. Majda, Introduction to PDEs and Waves for the Atmosphere and Ocean, Vol. 9 of Courant Lecture Notes, American Mathematical Society, Rhode Island, 2003.

[28] A.J. Majda and A. Bertozzi, Vorticity and Incompressible Flow, Cambridge University Press, Cambridge, England, 2001.

[29] A. Miranville, Lower bound on the dimension of the attractor for the Bénard problem with free surfaces, Nonlinear Analysis, vol.25, (1995), pp.1079-1094.

[30] A. Miranville and X. Wang, Robust exponential attractors to the Boussinesq system for convection at large Prandtl number, in preparation, 2005.

[31] A. Miranville and M. Ziane, On the dimension of the attractor for the Bénard problem with free surfaces, Russian Journal of Mathematical Physics, vol.5, no.4, (1998), pp. 489-502.

[32] P.H. Rabinowitz, Existence and non-uniqueness of regular solutions of the Bénard problem. Arch. Rational Mech. Anal., 29, 1968, 32-57.

[33] J. Robinson, Infinite Dimensional Dynamical System, Cambridge University Press, Cambridge, UK, 2001. 
[34] E. Siggia, High Rayleigh number convection. Annual review of fluid mechanics, Vol. 26, 137-168, Annual Reviews, Palo Alto, CA, 1994.

[35] R. Temam, Navier-Stokes equations and nonlinear functional analysis, 2nd ed. Philadelphia, Pa. : SIAM, 1995.

[36] R.M. Temam, Infinite Dimensional Dynamical Systems in Mechanics and Physics, 2nd ed, Springer-Verlag, New York, 1997.

[37] R. Temam, Navier-Stokes Equations, AMS Chelsea, Providence, Rhode Island, 2000.

[38] E.S. Titi and X. Wang, Small Darcy-Prandtl number convection in porous medium, in preparation, 2005.

[39] D.J. Tritton, 1988, Physical Fluid Dynamics, Oxford Science Publishing.

[40] X. Wang, Large Prandtl Number Limit of the Boussinesq System of Rayleigh-Bénard Convection, Applied Mathematics Letters, vol.17 (2004), 821-825.

[41] X. Wang, Infinite Prandtl Number Limit of Rayleigh-Bénard Convection, Communications on Pure and Applied Mathematics, vol 57, issue 10, (2004), 1265-1282.

[42] X. Wang, A Note on Long Time Behavior of Solutions to the Boussinesq System at Large Prandtl Number, Contemporary MathematICS, vol. 371, pp. 315-323, 2005.

[43] V.I. Yudovich, An example of the loss of stability and the generation of a secondary flow of a fluid in a closed container. Mat. Sb. (N.S.), 74, (116), 1967, 565-579. 